\title{
Factors to bear in mind regarding the use of dexmedetomidine
}

\author{
Jong-Yeon Park \\ Department of Anesthesiology and Pain Medicine, Asan Medical Center, University of Ulsan College of Medicine, \\ Seoul, Korea
}

In the Korean Journal of Anesthesiology (KJA), the first paper on dexmedetomidine was published in 2011; since then, more than 40 papers on this agent have been published in the KJA.

Dexmedetomidine is used in clinical practice as a sedative, analgesic, and sympatholytic. In one study, a single preanesthetic dose of dexmedetomidine $(0.5 \mu \mathrm{g} / \mathrm{kg})$ effectively suppressed hemodynamic responses to endotracheal intubation [1]. In another study, dexmedetomidine provided adequate sedation without respiratory depression [2]. Dexmedetomidine can be safely infused to decrease bleeding during surgery, and for smooth recovery from anesthesia [3]. Dexmedetomidine also prolongs the duration of spinal anesthesia or nerve block [4,5] and can prevent or attenuate opioid-induced hyperalgesia [6]. Recently, the applications of dexmedetomidine have become increasingly diverse. The current issue of the KJA includes both an interesting clinical study [7] and a case report [8] on dexmedetomidine use.

Choi et al. [7] compared the effects of dexmedetomidine versus remifentanil. In their study, dexmedetomidine (0.3-0.5 $\mu \mathrm{g} /$ $\mathrm{kg} / \mathrm{h}$ ) and remifentanil (at a target-effect site concentration of 2-3 ng/ml) were continuously infused, as an adjuvant to general anesthesia for thyroidectomy. The authors suggested that dexmedetomidine may be effective for the prevention of postoperative nausea and vomiting, and the postoperative analgesic effect of dexmedetomidine was also superior to that of remifentanil. Furthermore, the authors postulated that these results may have been due to a decreased noradrenergic activity as a result of $\alpha_{2}$ presynaptic inhibition in the locus coeruleus, or to a reduction in sympathetic outflow, which may in turn trigger postoperative nausea and vomiting.

Moon et al. [8] presented a case report entitled "Non-intubated thoracoscopic surgery for decortication of empyema under thoracic epidural anesthesia." In this case, video-assisted thoracoscopic surgery was performed in a non-intubated patient. During surgery, thoracic epidural anesthesia and target-controlled infusion of propofol and remifentanil were maintained. In addition, dexmedetomidine was continuously infused at a rate of $0.4 \mu \mathrm{g} / \mathrm{kg} / \mathrm{h}$. Although this regimen may appear somewhat reckless, the patient recovered fully without complications.

Taken together, these studies suggest that dexmedetomidine could be assistive in anesthesiologic practice in the near future. However, in addition to its possible benefits, certain drawbacks of dexmedetomidine must also be noted. First, bradycardia and hypotension could result from rapid infusion at higher doses, during or even after surgical operation. Furthermore, perioperative bradycardia and hypotension may be severe enough to require treatment. A final drawback of dexmedetomidine is delayed awakening; when it is used for a short surgery, delayed awakening and postoperative sedation could be problematic for anesthesiologists.

In summary, if anesthesiologists use dexmedetomidine only for carefully selected patients, they may find it helpful. However, dexmedetomidine may also produce effects that are problematic for anesthesiologists.

Corresponding author: Jong-Yeon Park, M.D., Ph.D.

Department of Anesthesiology and Pain Medicine, Asan Medical Center, University of Ulsan College of Medicine, 88, Olympic-ro 43-gil, Songpagu, Seoul 05505, Korea

Tel: 82-2-3010-3867, Fax: 82-2-3010-6958, Email: jongyeon_park@amc.seoul.kr

ORCID: http://orcid.org/0000-0003-0588-1903

Korean J Anesthesiol 2017 June 70(3): 233-234

https://doi.org/10.4097/kjae.2017.70.3.233

(c) This is an open-access article distributed under the terms of the Creative Commons Attribution Non-Commercial License (http://creativecommons.org/ licenses/by-nc/4.0/), which permits unrestricted non-commercial use, distribution, and reproduction in any medium, provided the original work is properly cited. 


\section{References}

1. Lee CW, Kim M. Effects of preanesthetic dexmedetomidine on hemodynamic responses to endotracheal intubation in elderly patients undergoing treatment for hypertension: a randomized, double-blinded trial. Korean J Anesthesiol 2017; 70: 39-45.

2. Kim SK, Song MH, Lee IJ, Lee JH, Choi IC. Dexmedetomidine for sedation in pediatric patients who received more than 20 sessions of radiation therapy: two cases report. Korean J Anesthesiol 2016; 69: 627-31.

3. Gupta K, Rastogi B, Gupta PK, Singh I, Singh VP, Jain M. Dexmedetomidine infusion as an anesthetic adjuvant to general anesthesia for appropriate surgical field visibility during modified radical mastectomy with i-gel ${ }^{\circledR}$ : a randomized control study. Korean J Anesthesiol 2016; 69: $573-8$

4. Lee MH, Ko JH, Kim EM, Cheung MH, Choi YR, Choi EM. The effects of intravenous dexmedetomidine on spinal anesthesia: comparision of different dose of dexmedetomidine. Korean J Anesthesiol 2014; 67: 252-7.

5. Song JH, Shim HY, Lee TJ, Jung JK, Cha YD, Lee DI, et al. Comparison of dexmedetomidine and epinephrine as an adjuvant to $1 \%$ mepivacaine in brachial plexus block. Korean J Anesthesiol 2014; 66: 283-9.

6. Lee C, Kim YD, Kim JN. Antihyperalgesic effects of dexmedetomidine on high-dose remifentanil-induced hyperalgesia. Korean J Anesthesiol 2013; 64: 301-7.

7. Choi EK, Seo Y, Lim DG, Park S. Postoperative nausea and vomiting after thyroidectomy: a comparison between dexmedetomidine and remifentanil as part of balanced anesthesia. Korean J Anesthesiol 2017; 70: 299-304.

8. Moon E, Go Y, Chung J, Yi J. Non-intubated thoracoscopic surgery for decortication of empyema under thoracic epidural anesthesia. Korean J Anesthesiol 2017; 70: 341-4. 